\title{
Small bowel adenocarcinoma: a cause of persistent iron deficiency anemia or abdominal discomfort
}

\author{
Kenta Hamada, Tomofumi Akasaka, Minoru Kato, Hiroyasu lishi \\ Osaka Medical Center for Cancer and Cardiovascular Diseases, Osaka, Japan
}

\begin{abstract}
A 74-year-old woman was referred to our hospital for management of persistent iron deficiency anemia with mild epigastric pain. Before referral, her esophagogastroduodenoscopy and colonoscopy findings had been normal, and she had been treated with oral iron preparation for the preceding 6 months. Physical examination and abdominal contrast-enhanced computed tomography at our institution were normal. Capsule endoscopy revealed an ulcerated jejunal lesion (Fig. 1). Double-balloon enteroscopy showed a subtotal circumferential irregular ulcerated jejunal lesion (Fig. 2). Biopsy specimens revealed moderately to poorly differentiated adenocarcinoma. Segmental jejunectomy with lymph node dissection was performed. The resected specimen showed pathological T4 adenocarcinoma without lymph node metastasis.

Small bowel adenocarcinoma is a rare cancer and is often detected late in its course because it is not amenable to conventional endoscopic examinations, such as esophagogastroduodenoscopy or colonoscopy, especially when it is distal to the duodenum [1]. Today, capsule endoscopy and balloon-assisted enteroscopy are available to examine the small intestine, and they have contributed to the detection of small bowel diseases. Small bowel cancer is, however, still difficult to diagnose because it is usually asymptomatic in the initial phase, while its clinical presentation is characterized by nonspecific abdominal discomfort, such as abdominal pain, nausea, vomiting, or gastrointestinal bleeding (both overt and occult) [2]. Capsule endoscopy, a less invasive examination than balloon-assisted enteroscopy, should be considered if esophagogastroduodenoscopy/colonoscopy and abdominal
\end{abstract}

Department of Gastrointestinal Oncology, Osaka Medical Center for Cancer and Cardiovascular Diseases, Osaka, Japan

Conflict of Interest: None

Correspondence to: Tomofumi Akasaka, Department of Gastrointestinal Oncology, Osaka Medical Center for Cancer and Cardiovascular Diseases, 1-3-3 Nakamichi Higashinari-ku, Osaka 537-8511, Japan, Tel.: +81 66972 1181, Fax: +81 66981 4067, e-mail: akasaka-to@mc.pref.osaka.jp

Received 06 December 2016; accepted 20 December 2016; published online 27 January 2017

DOI: https://doi.org/10.20524/aog.2017.0125

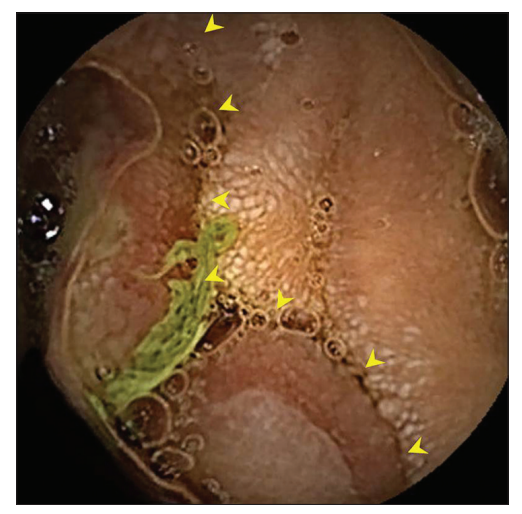

Figure 1 Capsule endoscopy revealed an ulcerated jejunal lesion (yellow arrowheads)

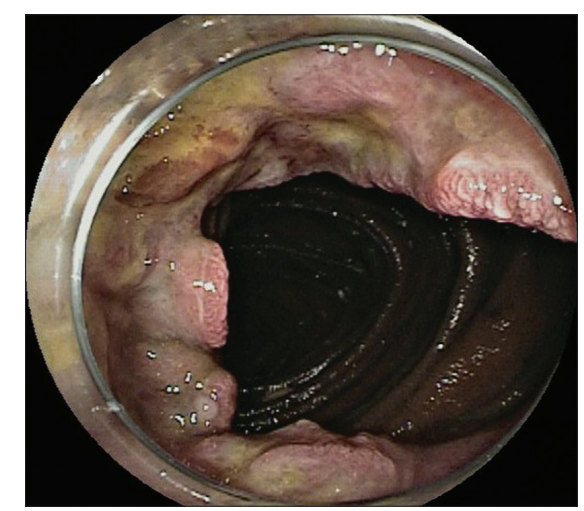

Figure 2 Double-balloon enteroscopy showed a subtotal circumferential irregular ulcerated jejunal lesion, diagnosed as adenocarcinoma on biopsy

computed tomography are normal, in the presence of persistent iron deficiency anemia or abdominal discomfort.

\section{References}

1. Dabaja BS, Suki D, Pro B, Bonnen M, Ajani J. Adenocarcinoma of the small bowel: presentation, prognostic factors, and outcome of 217 patients. Cancer 2004;101:518-526.

2. Li J, Wang Z, Liu N, Hao J, Xu X. Small bowel adenocarcinoma of the jejunum: a case report and literature review. World J Surg Oncol 2016;14:177. 